\title{
Coherent spin transport through helical edge states of topological insulator
}

\author{
R. A. Niyazov $\mathbb{D}^{1,2,3 凶}$, D. N. Aristov $\mathbb{D}^{1,2,3}$ and V. Yu. Kachorovskii $\mathbb{D}^{1,4}$
}

We study coherent spin transport through helical edge states of topological insulator tunnel-coupled to metallic leads. We demonstrate that unpolarized incoming electron beam acquires finite polarization after transmission through such a setup provided that edges contain at least one magnetic impurity. The finite polarization appears even in the fully classical regime and is therefore robust to dephasing. There is also a quantum magnetic field-tunable contribution to the polarization, which shows sharp identical Aharonov-Bohm resonances as a function of magnetic flux - with the period $h c / 2 e$-and survives at relatively high temperature. We demonstrate that this tunneling interferometer can be described in terms of ensemble of flux-tunable qubits giving equal contributions to conductance and spin polarization. The number of active qubits participating in the charge and spin transport is given by the ratio of the temperature and the level spacing. The interferometer can effectively operate at high temperature and can be used for quantum calculations. In particular, the ensemble of qubits can be described by a single Hadamard operator. The obtained results open wide avenue for applications in the area of quantum computing.

npj Computational Materials (2020)6:174; https://doi.org/10.1038/s41524-020-00442-z

\section{INTRODUCTION}

Quantum information processing attracts enormous interest in a broad scientific community ${ }^{1}$. Although the promise of quantum computers was recognized about 30 years ago, the real breakthrough in the creation of their key elements-networks of coherent spin qubits-was achieved only in the last decade ${ }^{2}$. The principal obstacle for further progress is connected with fast spin relaxation and dephasing, which prevents the creation of spin polarization and coherent spin transmission over long distances. Another challenging yet unsolved task of primary importance for information processing and quantum networking is all-electrical control of the electron spins ${ }^{3-5}$.

An effective low-cost room-temperature solution of these problems would allow for the tunable coherent transmission of the spin polarization over long distances. Ever since the proposal of spin field-effect transistor (SpinFET) ${ }^{6}$, numerous attempts to achieve coherent spin transmission and all-electrical manipulation by using setups of the various designs were unsuccessful ${ }^{7-13}$. In semiconductor devices, spin polarization usually originates from spin-orbit coupling and is never sufficiently large, in particular, due to the low efficiency of spin injection ${ }^{14}$. It can be somewhat increased by using non-electrical elements such as ferromagnetic contacts, which, however, dramatically deteriorate transport properties of the system. Furthermore, injected polarization rapidly decays due to spin relaxation processes.

In this article, we propose essential steps toward solving several critical problems of quantum information processing: spin filtering, long-distance spin transfer, and effective spin manipulation. Physically, spin filter blocks transmission of particles with one spin orientation, say spin-down, so that outgoing current acquires spin-up polarization. We introduce a method for the creation of spin-polarized electron beams based on using of helical edge states (HES) of two-dimensional (2D) topological insulator. Spin transport in HES was already discussed at zero temperature (see refs ${ }^{15-19}$ and references therein). Here, we demonstrate that, remarkably, the finite spin polarization arises at high temperature, even in the fully classical regime, and is therefore robust to dephasing.

The suggested method allows for $100 \%$ spin polarization and therefore has essential advantages over the existing approaches to spin filtering and spin transfer based on resonant tunneling diodes 20,21 , quantum $\operatorname{dots}^{22,23}, Y$ junctions ${ }^{24,25}$, and Aharonov-Bohm (AB) interferometers based on conventional materials ${ }^{26}$. In all these structures, spin polarization achieved so far was sufficiently small. More promising candidates for spin filtering are the quantum point contacts (QPC) with strong SO interaction and engineered structures incorporating QPC as building blocks ${ }^{27-32}$. Although the predicted spin polarization in QPC-based structures operating in the single-mode regime of SpinFET can be quite high ${ }^{30}$, one of the main problems in the way of coherent spin control-fast spin relaxation-remains unresolved. This implies that spin polarization cannot be transferred over a distance exceeding the spin relaxation length which is typically not quite large for conventional semiconductors with SO interaction.

Here, we study spin transport through the edge states of the topological insulator and show that the spin polarization can be transferred for large distances on the order of the edge state's length. This distance can be made even longer by building arrays of several HES. In contrast to all previous studies of spin-selective transport via HES, we find that large spin polarization can be created and transferred at high temperatures thus opening a wide avenue for application in quantum computing. In particular, we demonstrate that obtained results can be formulated in terms of a flux-tunable ensemble of qubits giving an equal contribution to charge and spin transport. Our study is a direct generalization of recent research on controlling quantum qubits by various types of interferometers and using them for quantum computing ${ }^{17,33-38}$.

\footnotetext{
${ }^{1}$ loffe Institute, 194021 St. Petersburg, Russia. ${ }^{2}$ Department of Physics, St. Petersburg State University, St. Petersburg 198504, Russia. ${ }^{3}$ NRC 'Kurchatov Institute', Petersburg Nuclear Physics Institute, Gatchina 188300, Russia. ${ }^{4}$ CENTERA Laboratories, Institute of High Pressure Physics, Polish Academy of Sciences, 01-142 Warsaw, Poland.

凶email: ramilniyazov@gmail.com
} 
In particular, it was predicted that conventional interferometers with spin-orbit (SO) interaction (or an array of such interferometers) can be used as one-qubit quantum gates of various types (X-gate, Z-gate, phase gate, and Hadamard gate) ${ }^{33}$. Such qubits can be controlled by changing the magnetic field and the strength of the SO interaction ${ }^{17,33}$. Taking into account, the electron-electron interaction makes it also possible to construct effective two-qubit computational schemes in two coupled interferometers based on conventional materials $\mathrm{s}^{35,36}$, on edge states of the integer quantum Hall effect $^{37,38}$ and 'on helical states $^{34}$. Signatures of electron-electron interaction in HES was already observed experimentally ${ }^{39,40}$.

The computational schemes, proposed so far, imply the control of so-called flying qubits with a given energy and can be directly applied at zero temperature. However, in realistic systems, the electrons enter the interferometer from thermalized contacts, which implies averaging within the temperature window around the Fermi energy. Since the phases accumulated by an electron passing through two arms of the interferometer are energydependent, the question arises whether thermal averaging violates the efficiency of the proposed computational schemes. This is exactly the question that we address in this work. We demonstrate that using tunneling interferometers based on HES allows one for the transfer of spin polarization at a large distances as well as quantum computing at high temperatures. We also find that the energy levels of almost closed interferometer form an ensemble of $T / \Delta$ qubits providing equal contributions into the spin and charge transport. This means that in HES-based setups, the interference survives thermal averaging (see ref. ${ }^{41}$ for discussion of this problem in conventional interferometers). Hence, using such interferometers might be a neat way to overcome the main problems of spin networking, namely, the sensitivity of spin polarization to dephasing and relaxation processes and the requirement of very low temperature.

\section{RESULTS}

Key idea

We propose to explore the unique properties of HES existing at the edges of 2D topological insulators, which are materials insulating in the bulk but exhibiting conducting channels at the surface or at the boundaries. In particular, the 2D topological insulator phase was predicted in $\mathrm{HgTe}$ quantum wells ${ }^{42,43}$ and confirmed by direct measurements of conductance of the edge states $^{44}$ and by the experimental analysis of the non-local transport $^{45-48}$. These states are one-dimensional helical channels where the electron spin projection is connected with its velocity, e.g., electrons traveling in one direction are characterized by spin "up", while electrons moving in the opposite direction are characterized by spin "down". Remarkably, the electron transport via HES is ideal, in the sense that electrons do not experience backscattering from conventional non-magnetic impurities, similarly to what occurs in edge states of Quantum Hall Effect systems, but without invoking high magnetic fields (for a detailed discussion of properties of HES, see refs ${ }^{49,50}$ ).

Hence, in the absence of magnetic disorder, the boundary states are ballistic and topologically protected from external perturbations. Due to this key advantage, a spin traveling along the edge does not relax, so that such states perfectly match the purposes of quantum spin networking. Importantly, even a nonmagnetic lead splits the incoming electron beam into two parts: right-moving electrons with spin-up and left-moving electrons with spin-down. If the transmission over one of the shoulders of the system is blocked, say, by inserting a strong magnetic impurity into the upper shoulder, then only the down shoulder remains active and the spin polarization of outgoing electrons can achieve $100 \%$. Remarkably, this mechanism is robust to dephasing and, therefore, works at high temperatures. We find a quantum contribution to polarization, which shows Aharonov-Bohm oscillations with the magnetic flux piercing the area encompassed by HES and is therefore tunable by the external magnetic field. This contribution survives at relatively high temperatures.

We also demonstrate that tunneling interferometer can be described in terms of an ensemble of flux-tunable qubits giving equal contributions to conductance and spin polarization. The number of active qubits participating in the charge and spin transport is given by the ratio of the temperature and the level spacing. The interferometer can effectively operate at high temperatures and can be used for quantum calculations. In particular, the ensemble of qubits can be described by a single flux-tunable Hadamard operator. Measurement of the conductance and the spin polarization is one of the ways to read out information about qubit states.

\section{Model}

The Hamiltonian of the edge is given by $H=\int d x\left(\mathcal{H}_{0}+\mathcal{H}_{\text {imp }}\right)$ with coordinate $x$ running along the edge. Here,

$$
\mathcal{H}_{0}=-i v_{\mathrm{F}}\left(\psi_{\uparrow}^{\dagger} \partial_{x} \psi_{\uparrow}-\psi_{\downarrow}^{\dagger} \partial_{x} \psi_{\downarrow}\right)
$$

is the unperturbed HES Hamiltonian with the Fermi velocity $v_{\mathrm{F}}$. For simplicity, we assume that the interferometer contains classical impurities with large magnetic moments $\mathbf{M}_{n},\left|\mathbf{M}_{n}\right|=M \gg 1$ (a small ferromagnetic island can serve as such an impurity), neglecting the feedback effect related to the dynamics of this moment caused by exchange interaction with the ensemble of right- and left-moving electrons (for infinite HES this effect was discussed in ref. ${ }^{51}$ ). Then, the isotropic exchange interaction with magnetic impurities located at points $x_{n}$ has the form

$\mathcal{H}_{\text {imp }}=g \sum_{n} \boldsymbol{\sigma} \mathbf{M}_{n} \delta\left(x-x_{n}\right)$,

where $g$ is the coupling constant and $\mathbf{M}_{n}=$ $M\left(\sin \eta_{n} \cos \varphi_{n}, \sin \eta_{n} \sin \varphi_{n}, \cos \eta_{n}\right)$. Here angles $\eta_{n}$ and $\varphi_{n}$ describe the direction of $\mathbf{M}_{n}$.

In the general case, the edge contains randomly distributed magnetic impurities shown by dots in Fig. 1. However, as we demonstrate below, the simplest case of an interferometer containing a single impurity captures basic physics of the problem. At the same time, this case is the most realistic, since we discuss non-magnetic materials. Hence, we start with a discussion of the interferometer with the single impurity placed in the upper shoulder. By using Eq. (2), one can find the scattering matrix of this impurity

$\hat{S}_{M}=\left(\begin{array}{cc}e^{i \alpha} \cos \theta & i \sin \theta e^{-i \varphi} \\ i \sin \theta e^{i \varphi} & e^{-i a} \cos \theta\end{array}\right)$,

where $a$ is the forward scattering phase, and $\sin ^{2} \theta$ is the backscattering probability (previously, we, for simplicity, considered the model of impurity with zero forward scattering phase,

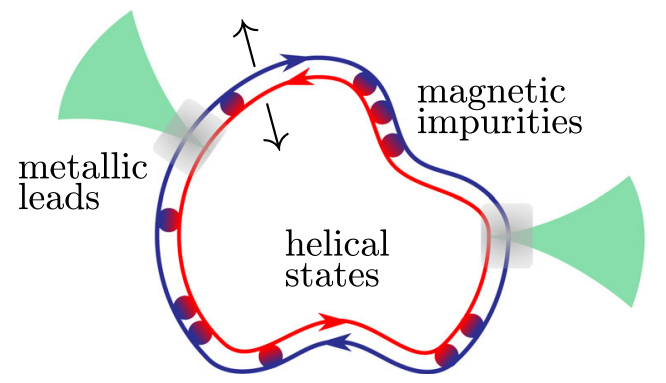

Fig. 1 Setup. Helical edges state tunnel-coupled to the metallic point contacts. The magnetic impurities are marked by dots. 

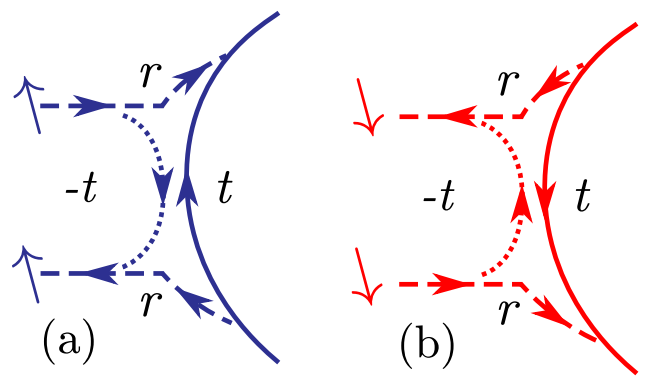

Fig. 2 Point tunneling contact. Non-magnetic point contact between the helical ring and the spinful wire. Different spin projections (shown by blue (a) and red color (b)) do not mix at the contact and correspond to electrons propagating along the helical edge in the opposite directions. Scattering on this contact is described by the spin-independent real amplitudes $t$ and $r$ obeying $t^{2}+r^{2}=1$

$a=0^{52}$ ). For weak impurity with $\rho_{0}=g M / v_{\mathrm{F}} \ll 1$, one gets: $a \approx$ $\rho_{0} \cos \eta \ll 1$ and $\theta \approx \rho_{0} \sin \eta \ll 1$.

The spin transport through HES of a $2 \mathrm{D}$ topological insulator assumes tunnel coupling to leads (see Fig. 1). The tunneling conductance of this setup is given by $G=2 \times\left(e^{2} / h\right) \mathcal{T}$, where factor 2 corresponds to two conducting channels. For the case of spin-unpolarized contacts, the transmission coefficient, $\mathcal{T}$, can be represented as an average over incoming spin polarizations $\mathcal{T}=$ $\left(\mathcal{T}_{\uparrow}+\mathcal{T}_{\downarrow}\right) / 2$. Here, $\mathcal{T}_{\beta}=\sum_{\alpha} T_{\alpha \beta}=\sum_{\alpha}\left\langle\left|t_{\alpha \beta}(\epsilon)\right|^{2}\right\rangle_{\epsilon^{\prime}} a, \quad \beta=\uparrow, \downarrow$, $\langle\cdots\rangle_{\epsilon}=-\int d \epsilon(\cdots) \partial_{\epsilon} f_{\mathrm{F}}(\epsilon), f_{\mathrm{F}}(\epsilon)$ is the Fermi function and $t_{\alpha \beta}$ is a spindependent transition amplitude. The spin polarization of outgoing electrons reads $P_{z}=\left(T_{\uparrow \uparrow}+T_{\uparrow \downarrow}-T_{\downarrow \uparrow}-T_{\downarrow \downarrow}\right) / 2 \mathcal{T}$. For the case $T_{\uparrow \downarrow}=T_{\downarrow \uparrow}$ (see Eq. (9) below), this equation simplifies

$P_{z}=\frac{\mathcal{T}_{\uparrow}-\mathcal{T}_{\downarrow}}{\mathcal{T}_{\uparrow}+\mathcal{T}_{\downarrow}}$,

where $z$ axis coincides with the direction of spin at the position of outgoing contact.

We consider non-magnetic leads, thus assuming that different spin projections do not mix at the tunneling contacts, so that electrons entering the edge with opposite spins move in the opposite directions (see Fig. 2). Such contacts are characterized by spin-independent amplitudes $r$ and $t$, obeying $|t|^{2}+|r|^{2}=1$. We assume that $t$ and $r$ are real and positive and parameterize them as follows: $r=\sqrt{1-e^{-2 \lambda}}, t=e^{-\lambda}, 0<\lambda<\infty$ (parameter $\lambda$ is connected with tunneling transparency $\gamma$ used in ref. ${ }^{52}$ as follows: $\gamma=\tanh (\lambda / 2))$

We will study both classical and quantum contributions to the spin polarization. The quantum contribution is sensitive to the magnetic field due to the $A B$ effect. Hence our setup represents an example of $A B$ interferometer built on HES. The form and shape of the $A B$ oscillations strongly depend on the relation between temperature $T$ and level spacing $\Delta=2 \pi v_{\mathrm{F}} / L$, which is controlled by total interferometer circumference $L$ and the Fermi velocity $v_{\mathrm{F}}$. Let us do some estimates. For $L=10 \mu \mathrm{m}$ and $v_{\mathrm{F}}=10^{7} \mathrm{~cm} \mathrm{~s}^{-1}$, we get $\Delta \approx 3 \mathrm{~K}$. As seen from this estimate, the case

$T \gg \Delta$

is much more interesting for possible applications. We will focus on this case throughout the article. There is also an upper limitation for temperature. For good quantization, $T$ should be much smaller than the bulk gap of the topological insulator: $T \ll \Delta_{\mathrm{b}}$. For the first time, quantum spin Hall effect was observed in structures based on $\mathrm{HgTe} / \mathrm{CdTe}^{53}$ and $\ln \mathrm{As} / \mathrm{GaSb}^{54}$, which had a rather narrow bulk gap, $<100 \mathrm{~K}$. Substantially large values were observed recently in $\mathrm{WTe}_{2}$, where gap of the order of $500 \mathrm{~K}$ was observed $^{55}$, and in bismuthene grown on a SiC (0001) substrate, where a bulk gap of about $0.8 \mathrm{eV}$ was demonstrated ${ }^{5,57}$ (see also recent discussion in ref. ${ }^{39}$ ). Thus, recent experimental studies unambiguously indicate the possibility of transport through HES at room temperature, when the condition $\Delta_{\mathrm{b}} \gg T \gg \Delta$, needed for applicability of our theory, can be easily satisfied. Importantly, this condition ensures the universality of spin and charge transport (see discussion in ref. ${ }^{52}$ ), which do not depend on details of the systems, in particular, on the device geometry.

Tunneling conductance

Recently, we discussed dependence of the tunneling conductance $G$ of such a setup on the external magnetic flux $\Phi$ piercing the area encompassed by edge states ${ }^{52}$. For consistency, we briefly summarize main results of ref. ${ }^{52}$ here. We have demonstrated the existence of interference-induced effects, which are robust to the temperature, i.e., survive under the condition Eq. (5), and can therefore be obtained for relaxed experimental conditions (for discussion of this regime in conventional interferometers, see refs $\left.{ }^{41,58-61}\right)$. Specifically, we have found that $G$ is structureless in ballistic case but shows periodic dependence on dimensionless flux $\phi=\Phi / \Phi_{0}$ (here, $\Phi_{0}=h c / e$ is the flux quantum), with the period $1 / 2$, in the presence of a single magnetic impurity in one of the interferometer's shoulders. Such a weak impurity can be taken into account perturbatively provided that $\theta \ll \max (\lambda, 1)$. The resulting analytical expression for the transmission coefficient reads $^{52}$

$$
\mathcal{T}=\tanh \lambda-\frac{\tilde{\theta}^{2}}{2} \tanh ^{2} \lambda
$$

where $\tilde{\theta}^{2}=\theta^{2}\left(1+\left.\mathcal{C}\right|_{\theta=0}\right)$ and

$$
\mathcal{C}=\frac{t^{4} e^{4 i \pi \phi}}{1-t^{4} \cos ^{2} \theta e^{4 i \pi \phi}}+\frac{t^{4} e^{-4 i \pi \phi}}{1-t^{4} \cos ^{2} \theta e^{-4 i \pi \phi}}
$$

represent "ballistic Cooperon"52 which is the interference contribution of the processes, in which the electron wave splits at the impurity into two parts passing the setup in the opposite directions and returning to impurity after a number of revolutions with equal winding numbers (see Fig. 6 of ref. ${ }^{52}$ ). The factor

$\frac{\tilde{\theta}^{2}}{\theta^{2}}=1+\left.\mathcal{C}\right|_{\theta=0}=\frac{\sinh (4 \lambda)}{\cosh (4 \lambda)-\cos (4 \pi \phi)}$

describes the coherent enhancement of backscattering probability caused by multiple returns to the impurity. This enhancement has a purely quantum nature. The classical limit, when all interference processes are neglected, can be obtained by averaging $\mathcal{T}$ over flux. Having in mind that $\langle\mathcal{C}\rangle_{\phi}=0$, we find that "classical" conductance is given by Eq. (6) with the replacement $\tilde{\theta}^{2} \rightarrow \theta^{2}$. Hence, in the perturbative regime, $\mathcal{T}$ obeys $1 / 2$-flux periodicity $\mathcal{T}(\phi+1 / 2)=\mathcal{T}(\phi)$ and shows sharp identical antiresonances at integer and half-integer values of $\phi$ in the limit of weak tunneling coupling, $\lambda \ll 1$. In the latter limit, the non-perturbative effects lead to the appearance of the additional contribution $2 \theta^{2}$ in the denominator of Eq. (8) ${ }^{52}$. Physically, this corresponds to the broadening of the antiresonances because of multiple coherent scattering events.

\section{Spin polarization}

Next, we discuss the spin polarization of outgoing electrons. We will limit ourselves to the discussion of non-interacting electrons focusing on the high-temperature cases. For discussion of spin polarization in the case of the low temperature, see refs ${ }^{62-66}$, while interaction-induced ${ }^{67}$ and quantum pumping generated ${ }^{68}$ spin currents were considered for Fabry-Pérot geometry at $\phi=0$. We will demonstrate that the finite polarization appears even in the fully classical regime and therefore robust to dephasing. There also exists quantum contribution to polarization which survives at relatively large temperatures and is tunable by magnetic flux 


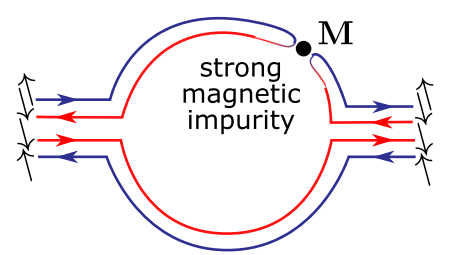

Fig. 3 Single strong impurity. Strong magnetic impurity blocks the transmission of one component of the electron spin. For open setup, $\lambda=\infty$, this leads to $100 \%$ polarization. Polarization reverses sign when a strong impurity is moved from upper to lower shoulder.

piercing the interferometer. Specifically, we will demonstrate that similar to tunneling conductance, the quantum contribution to the polarization shows sharp identical resonances as a function of magnetic flux with maxima (in the absolute value) at integer and half-integer values of the flux.

In order to illustrate our approach, we consider a single impurity placed in the upper shoulder of the interferometer and discuss a simple limiting case: $\lambda=\infty, \theta=\pi / 2$ (strong impurity, open interferometer). In this case, $t=0$ and $r=1$, so that electrons with spin-up (down) can go only through the upper (lower) shoulder of the interferometer (see Fig. 2). On the other hand, probability of backscattering by the impurity is given by $\sin ^{2} \theta=1$, so that the impurity fully blocks transmission through the upper shoulder (see Fig. 3). Hence, such a setup serves as an ideal spin filter: the transmission of electrons with spin-up is blocked while spin-down electrons can freely pass through the interferometer. Consequently, the outgoing polarization reaches $100 \%$. Evidently, this is a classical result that is not sensitive to dephasing. At the same time, a fully polarized electron beam corresponds to a pure quantum spin state. In other words, even in the classical regime, the interferometer can create pure quantum states within the discussed limiting case. Below, we present detailed calculations of the spin polarization for a number of other cases.

Results of ref. ${ }^{52}$ can be easily generalized for calculation of spin polarization. For a weak impurity placed in the upper shoulder of interferometer, direct summation of amplitudes in a full analogy with ref. ${ }^{52}$ yields in the lowest order in $\theta^{2}$ :

$T_{a \beta}=\delta_{\alpha \beta} \tanh \lambda-\frac{a \beta \exp [\lambda(a+\beta)]}{4 \cosh ^{2} \lambda} \tilde{\theta}^{2}$

where $a, \beta= \pm 1$, for a spin-up and -down, respectively. Classical probabilities $\left\langle T_{\alpha \beta}\right\rangle_{\phi}$ are given by Eq. (9) with the replacement $\tilde{\theta}^{2} \rightarrow \theta^{2}$.

The perturbative in $\theta^{2}$ spin polarization can be found from Eqs. (4) and (9):

$$
P_{z}=-\frac{\tilde{\theta}^{2}}{2}=-\frac{\theta^{2}}{2} \frac{\sinh (4 \lambda)}{\cosh (4 \lambda)-\cos (4 \pi \phi)} .
$$

As is seen from this equation, polarization shows sharp identical antiresonances at integer and half-integer values of flux for weak tunneling coupling, $\lambda \ll 1$, and weak $A B$ oscillations for almost open setup, $\lambda \gg 1$. The analogous calculation for a single impurity with the same strength, $\theta$, placed in the lower shoulder of the interferometer yields Eq. (10) with the opposite sign. In the classical regime, the polarization is simply given by $P_{z}= \pm \theta^{2} / 2$, with the sign determined by the position of impurity. One can follow the evolution of polarization from quantum to classical case by introducing a dephasing process with the rate $\Gamma_{\varphi}$ which suppresses "ballistic Cooperon". Technically, this means replacement $\lambda \rightarrow \lambda+\lambda_{\varphi}$ in Eq. (10), where $\lambda_{\varphi}=\pi \Gamma_{\varphi} / 2 \Delta$ (see ref. ${ }^{52}$ ). For $\lambda_{\varphi} \rightarrow \infty$, we restore the classical result. Away from the resonant points (more precisely, for $\cos (4 \pi \phi)<0$ ), dephasing leads to the increase of polarization because the interference for such values of $\phi$ is destructive.
The microscopical calculation of $\Gamma_{\varphi}$ in HES is a non-trivial question. In conventional systems, including infinite singlechannel quantum wires, dominates dephasing caused by electron-electron scattering. In HES, such dephasing is suppressed for the same reason as ordinary impurity backscattering. Nonzero (very slow) dephasing due to electron-electron interaction arises only when Rashba-type terms are present (see also Supplementary Note 1) and slow energy dependence of these terms on energy is taken into account ${ }^{69,70}$. Additional suppression of the interactioninduced dephasing is expected due to finite geometry of the setup similar to the case of conventional single-channel interferometers ${ }^{41}$. A very slow dephasing occurs due to the dynamics of the magnetic impurity. Such dynamics can arise due to the interaction directly with the conduction electrons $s^{51}$ and due to the presence of a magnetic bath ${ }^{52}$. In the latter case, assuming that the averaged magnetic moment of impurity relaxes as $\langle\mathbf{M}(0) \mathbf{M}(t)\rangle=M^{2} \exp \left(-\Gamma_{0} t\right)$ one gets $\Gamma_{\varphi}=\Gamma_{0}^{52}$. Importantly, all proposed mechanisms lead to a dephasing rate significantly slower (at least in the framework of theoretical models) than in conventional systems.

Let us now consider a setup with a number of randomly distributed impurities. We start our discussion with the classical regime $\left(\lambda_{\varphi} \rightarrow \infty\right)$. One finds then $T_{\alpha \beta}$ as the sum over contributions from classical trajectories propagating clockwise and counterclockwise and experiencing collisions by magnetic impurities with forward probability $\cos ^{2} \theta$ and backward probability $\sin ^{2} \theta$. Relations between classical currents flowing from different sides of the impurity read: $J_{n+1}^{\uparrow}=\cos ^{2} \theta J_{n}^{\uparrow}+\sin ^{2} \theta J_{n+1}^{\downarrow}, J_{n}^{\downarrow}=\sin ^{2} \theta J_{n}^{\uparrow}+$ $\cos ^{2} \theta J_{n+1}^{\downarrow}$. The vectors $\mathbf{J}_{n}=\left(J_{n}^{\uparrow}, J_{n}^{\downarrow}\right)$ and $\mathbf{J}_{n+1}=\left(J_{n+1}^{\uparrow}, J_{n+1}^{\downarrow}\right)$ are thus connected by the classical transfer matrix

$$
\hat{W}_{\mathrm{cl}}(\theta)=1+\tan ^{2} \theta \hat{P}, \hat{P}=\left(\begin{array}{ll}
-1 & 1 \\
-1 & 1
\end{array}\right) \text {. }
$$

It obeys simple multiplication rule, $\hat{W}_{\mathrm{cl}}\left(\theta_{1}\right) \hat{W}_{\mathrm{cl}}\left(\theta_{2}\right)=\hat{W}_{\mathrm{cl}}(\theta)$, $\tan ^{2}(\theta)=\tan ^{2}\left(\theta_{1}\right)+\tan ^{2}\left(\theta_{2}\right)$. Let us consider a setup containing $N_{\mathrm{u}}$ impurities in the upper shoulder, characterized by $\theta_{1}, \ldots, \theta_{N_{\mathrm{u}}}$ and $N_{\mathrm{l}}$ in the lower one characterized by $\theta_{1}^{\prime}, \ldots, \theta_{N_{u}}^{\prime}$. Due to multiplicativity property, one can equivalently consider a setup with two impurities having effective strengths $g_{\mathrm{u}}=\sum_{n=1}^{N_{\mathrm{u}}} \tan ^{2}\left(\theta_{n}\right)$, and $g_{\mathrm{l}}=\sum_{n=1}^{N_{\mathrm{l}}} \tan ^{2}\left(\theta_{n}^{\prime}\right)$, placed, respectively, in the upper and lower shoulder of the interferometer. Next, we assume that the current entering the interferometer from the left contact is unpolarized, and use the scattering probabilities $r^{2}$ and $t^{2}$ to write balanced equations for currents at the left and right contacts. We find

$$
P_{z}=\frac{g_{\mathrm{l}}-g_{\mathrm{u}}}{2+\left(g_{\mathrm{u}}+g_{\mathrm{l}}\right) \operatorname{coth} \lambda} .
$$

Hence, the finite polarization exists even in the classical regime and is therefore robust to dephasing. One can show that the conductance in the classical regime looks

$$
\mathcal{T}=\frac{g_{\mathrm{u}}+g_{\mathrm{l}}+2 \tanh \lambda}{2\left[1+g_{\mathrm{u}} g_{\mathrm{l}}+\left(g_{\mathrm{u}}+g_{\mathrm{l}}\right) \operatorname{coth} 2 \lambda\right]} .
$$

The above perturbative analysis of a single impurity case shows that all quantum effects are encoded in the renormalization of backscattering probability: $\theta^{2} \rightarrow \tilde{\theta}^{2}$. Physically, it happens because such effects arise due to the interference of multiple returns to magnetic impurity along the ballistic trajectories propagating in opposite directions and having the same winding numbers. Therefore, generalization for the case of many impurities is trivial: one should expand Eq. (12) over impurities backscattering probabilities in lowest order and take into account the renormalization, Eq. (8). For the case of weak impurities of equal strength, we find that $\mathcal{T}$ is given by Eq. (6) with the replacement 


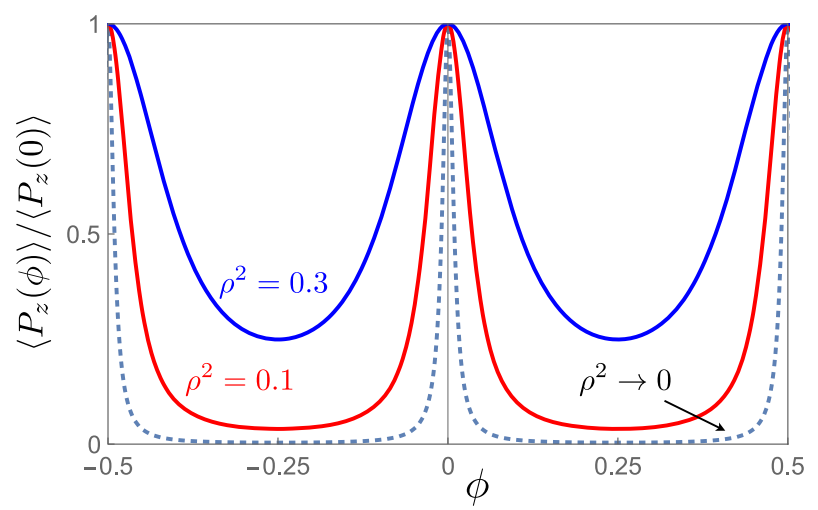

Fig. 4 Resonances in the polarization. Broadening of resonances in polarization with increasing strength of magnetic disorder, $\rho^{2}=\left(N_{u}+N_{l}\right) \rho_{0}^{2} / 3$, and $\lambda=0.03$.

$\tilde{\theta}^{2} \rightarrow \tilde{\theta}^{2}\left(N_{\mathrm{u}}+N_{\mathrm{l}}\right)$, and the polarization reads

$P_{z}=\frac{\tilde{\theta}^{2}\left(N_{\mathrm{I}}-N_{\mathrm{u}}\right)}{2} \underset{\lambda \ll 1}{\longrightarrow} \frac{2 \lambda \theta^{2}\left(N_{\mathrm{l}}-N_{\mathrm{u}}\right)}{1-\cos (4 \pi \phi)+8 \lambda^{2}}$.

One can generalize this formula in order to take into account nonperturbative effects with respect to impurity strength (still assuming $\theta<1)$. Corresponding calculations are presented in Supplementary Note 2. The result is shown in Fig. 4. As seen, nonperturbative effects lead to the broadening of the resonances.

One of the most important conclusions of this section is the universality of obtained results which was discussed previously in the context of conductance calculation ${ }^{52}$. The final equation for polarization is not sensitive to the geometry of the device and details of the structure. Also, the Berry phase drops out from the final result. Physically, this happens due to our assumption $T \gg \Delta$. In this case, quantum contribution to the conductance depends on quantum return probability (ballistic Cooperon) which is the universal quantity.

\section{Ensemble of qubits}

The transport through a HES-based interferometer was examined above (and earlier in ref. ${ }^{52}$ ) by a direct summation of the amplitudes of quantum transitions. Equivalently, the charge transfer through the interferometer can be viewed as tunneling through an ensemble of equivalent qubits.

The latter approach is applicable for the important cases of either $\phi \ll 1$ or $\phi-1 / 2 \ll 1$ and weak impurities. Although it does not allow one to describe transmission coefficient and polarization for $\phi \sim 1$, it is more illustrative physically and much more suitable for the analysis of quantum computing in the system under discussion. Below, we discuss this approach for the case if the interferometer with a single magnetic impurity.

The key idea is that the tunneling amplitude through the interferometer can be presented as a sum of the transition amplitudes through intermediate states corresponding to quasistationary levels of an almost closed HES (similar approach for nonhelical single-channel interferometer was discussed in ref. ${ }^{59}$ ). As a starting point, we consider an interferometer in the limit of an infinitely weak tunnel coupling, i.e., a system of two closed HES. In the absence of magnetic impurity, quantum levels are given by the following formula, $\epsilon_{n}^{ \pm}(\phi)=\Delta(n \pm \phi)$, and for integer and halfinteger values of the flux, the level system is degenerate: $\epsilon_{n}^{+}(0)=\epsilon_{n}^{-}(0), \epsilon_{n}^{+}(1 / 2)=\epsilon_{n+1}^{-}(1 / 2)$. Magnetic impurities lift this degeneracy. In particular, for a single magnetic impurity described by Eq. (3), quantum levels are given by

$\epsilon_{n}^{ \pm}=\Delta\left(n \pm \phi_{0}\right)$,
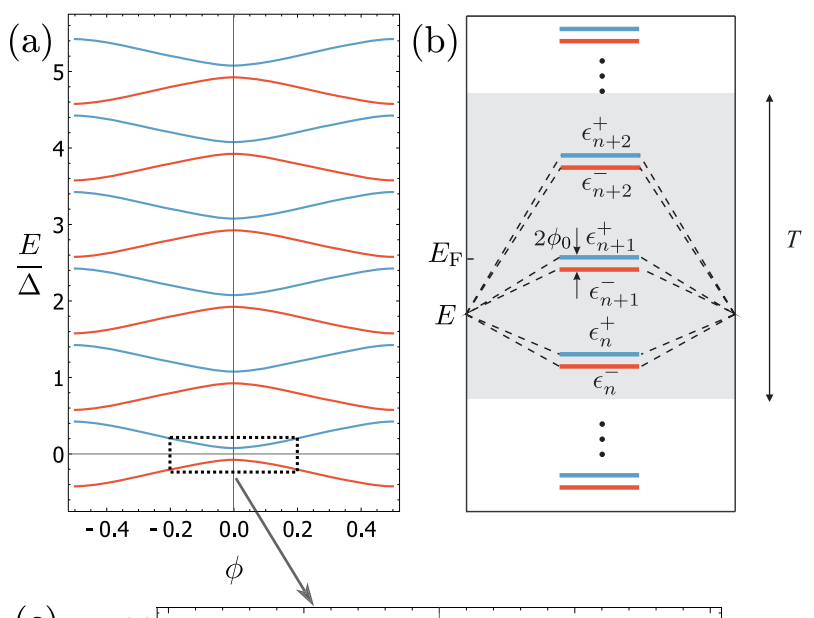

(c)

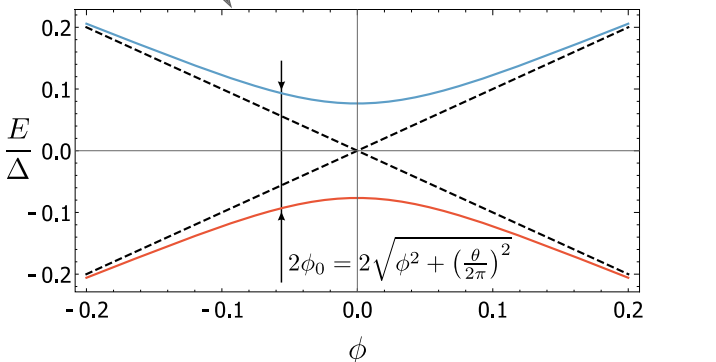

Fig. 5 Energy levels. a Energy levels of right- and left-moving electrons (red and blue curves, respectively) in the closed interferometer. b Transmission of the electrons through an ensemble of $T / \Delta$ active qubits. c Anticrossing at $\phi=0$.

where $\phi_{0}$ obeys

$\cos \left(2 \pi \phi_{0}\right)=\cos \theta \cos (2 \pi \phi)$

hence, anticrossing at $\phi=0$ and $\phi=1 / 2$. The energy levels are plotted in Fig. 5a, b. For weak impurity, splitting at anticrossing points, $\left.\left(\epsilon^{+}-\epsilon^{-}\right)\right|_{\phi=0}=2 \Delta \theta$, is small.

Details of calculations are presented in the "Methods" section. The form of wave functions shows that spinors corresponding to different $n$ have the same direction of local spins at the impurity position:

$\mathbf{S}^{a}\left(x_{0}\right)=-\mathbf{S}^{-a}\left(x_{0}\right)=\frac{1}{2}\left\langle\psi_{n}^{a}\left(x_{0}\right)|\hat{\boldsymbol{\sigma}}| \psi_{n}^{a}\left(x_{0}\right)\right\rangle, a= \pm$.

With increasing $x$ starting from $x=x_{0}+0$, the $z$ component of local spin does not change, $S_{z}^{a}(x)=S_{z}^{a}\left(x_{0}\right)$. By contrast, the perpendicular component of local spin rapidly rotates, rotating by angle $4 \pi\left(n \pm \phi_{0}\right)$ upon arrival to the point $x=x_{0}-0$ after the passage of the ring.

Anticrossing at $\phi=0$ is illustrated in Fig. $5 c$ (picture at $\phi=1 / 2$ is fully analogous). For weak impurity, in the vicinity of anticrossing point, we have

$$
2 \pi \phi_{0} \approx \sqrt{(2 \pi \phi)^{2}+\theta^{2}},
$$

and, consequently,

$\delta \epsilon=\epsilon_{n}^{+}-\epsilon_{n}^{-} \approx 2 \Delta \sqrt{\phi^{2}+(\theta / 2 \pi)^{2}}$,

for $\theta \ll 1, \phi \ll 1$. As seen, close to anticrossing points the distance between $(n,+)$ and $(n,-)$ is small, so levels are almost degenerate, and can be controlled either by the perpendicular magnetic field, which affects both $\phi$ and $\theta$, or by parallel field, which also rotates moment of the magnetic impurity thus changing $\theta$. Close to points $\phi=0$ and $\phi=1 / 2, z$ component of spin changes very 
6

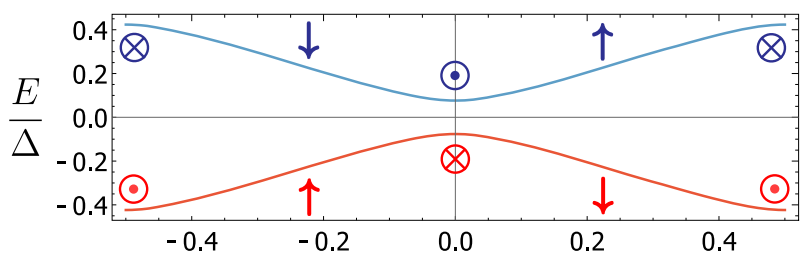

(a)

$\phi$

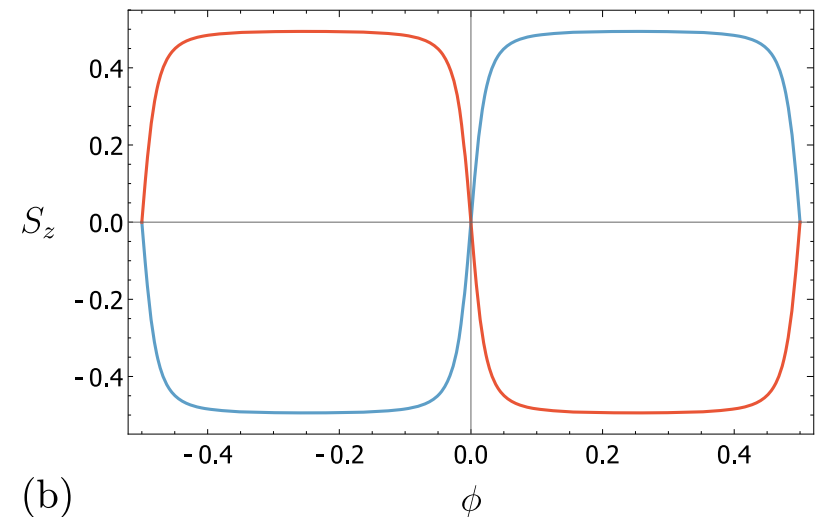

Fig. 6 Qubit control. a Variation of qubit states with the magnetic flux. b For $\phi=0$ both right- and left-moving electrons (red and blue curves, respectively) have spins perpendicular to the $z$ axis. Electron spin switches between $S_{z}=1$ to $S_{z}=-1$ within a narrow interval of $\phi$.

sharply (see Fig. 6)

$S_{z}^{ \pm} \approx \mp \frac{\pi \phi}{\sqrt{(2 \pi \phi)^{2}+\theta^{2}}}$, for $|\phi| \ll 1, \theta \ll 1$,

and similarly for $|\phi-1 / 2| \ll 1$.

For week tunneling coupling $(\lambda \ll 1)$, the tunneling transport of the electrons through the interferometer can be described in terms of transmission amplitudes (see ref. ${ }^{59}$ and "Methods" section)

$A_{n}^{a}(\epsilon) \propto \frac{1}{\epsilon-\epsilon_{n}^{a}-i \Gamma / 2}+\ldots$,

where $\Gamma \approx 2 \Delta \lambda / \pi$ is the tunneling rate, and $+\ldots$ stands for nonsingular contribution. Both $\mathcal{T}$ and $P$ can be expressed in terms of energy-averaged bilinear combinations of these amplitudes. There are "classical" terms, $\propto\left\langle\left|A_{n}^{\alpha}(\epsilon)\right|^{2}\right\rangle_{\epsilon}$, and interference terms, $\propto$ $\left\langle A_{n}^{a}(\epsilon) A_{m}^{\beta *}(\epsilon)\right\rangle_{\epsilon}$ with $(n, a) \neq(m, \beta)$, corresponding to transitions through different quantum levels (see Fig. $5 b$ ). For the case under discussion, $\lambda \ll 1, T \gg \Delta$, the interference contribution is dominated by terms with $n=m$ and $\beta=-a$,

$$
\left\langle A_{n}^{a}(\epsilon) A_{n}^{-a *}(\epsilon)\right\rangle_{\epsilon} \approx \frac{2 \pi i}{a \delta \epsilon+i \Gamma}\left(-\frac{\partial f_{\mathrm{F}}}{\partial \epsilon}\right)_{\epsilon=n \Delta},
$$

while interference processes with $n \neq m$ are described by a similar equation which contains the term $(n-m) \Delta \gg \delta \epsilon$ in the denominator and therefore is small.

\section{Quantum computing by qubit ensemble}

It is known that conventional interferometers with spin-orbit (SO) interaction (or an array of such interferometers) can be used as one-qubit quantum gates of various types (X-gate, Z-gate, phase gate, and Hadamard gate $)^{33}$, which manipulate spin states of the electrons with given energy-the so-called flying qubits. The flying qubits can be used for quantum calculations at very low temperatures $<100 \mathrm{mK}^{36}$. Analyzing analytical expression for energy- and spin-dependent transmission amplitudes $t_{\alpha \beta}(\epsilon)$ (see
Eq. (33) of the "Methods" section) one can-in a full analogy with ref. ${ }^{33}$-introduce quantum gates of different types. However, here we would like to focus on a different issue, namely, the possibility of high-temperature qubit manipulation. Since we consider an almost closed tunneling interferometer, we will use the language of the quantum levels introduced in the previous section.

The almost degenerate pairs of levels represent an ensemble of qubits with equal interlevel distance. The number of active qubits, which are able to participate in the spin and charge transport is given by

$\mathcal{N}_{\text {active }} \simeq \frac{T}{\Delta}$.

Transmission of charge and spin through the interferometer can be considered in terms of coherent hopping through these qubits (analogously to the case of conventional interferometer ${ }^{59}$ ) as illustrated in Fig. 5b.

Technically, in order to describe transition though qubit levels one should introduce projection operators $\hat{P}_{1}$ and $\hat{P}_{2}$ (see "Methods" section), which can be presented as

$P_{1,2}=\frac{1}{2}(1 \pm \hat{H})$.

Here, we introduced Hadamard operator

$\hat{H}=\left(\begin{array}{cc}a & b e^{-i \xi} \\ b e^{i \xi} & -a\end{array}\right)$,

where coefficients $b=e^{-2 \pi i \phi} \tan \theta / \sin \left(2 \pi \phi_{0}\right)$ and $a=$ $i\left[e^{-2 \pi i \phi} / \cos \theta-\cos \left(2 \pi \phi_{0}\right)\right] / \sin \left(2 \pi \phi_{0}\right)$, obey $a^{2}+b^{2}=1$ and depend on the strength of the impurity and the magnetic flux only, while the dependence on the energy is encoded in the exponents $e^{ \pm i \xi}$ entering off-diagonal terms of $\hat{H}$. The operator $\hat{H}$ has standard properties

$\hat{H}^{2}=1, \operatorname{Tr} \hat{H}=0$, det $\hat{H}=-1$.

Importantly, $\hat{H}$ can be tuned by the external magnetic field.

Off-diagonal elements of $\hat{H}$ rapidly oscillate with energy and, strictly speaking, one could introduce a set of Hadamard operators corresponding to different quantum levels in the interferometer: $\hat{H}_{n a}=\hat{H}_{\epsilon=\epsilon_{n a}}$. However, the results of direct calculations for conductance and spin polarization show that the dependence on $n$ drops out. Hence, we have an ensemble of qubits, which give coherent contributions to the charge and spin transport.

For $\theta \ll 1, \lambda \ll 1$, and $\phi \ll 1$, the transmission coefficient and polarization are expressed in terms of $\hat{H}$ as follows (see "Methods" section)

$\mathcal{T} \approx \frac{\pi \Gamma}{8 \Delta} \operatorname{Tr}(\hat{A}), \quad P_{z} \approx \frac{\pi \Gamma}{8 \Delta \mathcal{T}} \operatorname{Tr}\left(\hat{\sigma}_{z} \hat{A}\right)$

where $\Gamma=4 \lambda v_{F} / L=2 \lambda \Delta / \pi$ is the tunneling rate and

$\hat{A}=\frac{\hat{S}(\Gamma+i \delta \epsilon \hat{H})^{\dagger} \hat{S}(\Gamma+i \delta \epsilon \hat{H})}{\Gamma^{2}+\delta \epsilon^{2}}+\frac{2 \pi \Gamma}{\Delta} \hat{\sigma}_{z}$,

where information about tunneling coupling is encoded in $\Gamma$ and in the matrix

$\hat{S}=\left(\begin{array}{cc}e^{-\lambda} & 0 \\ 0 & e^{\lambda}\end{array}\right)$.

Hence, measurement of $\mathcal{T}$ and $P_{z}$ allows one to read out information about the ensemble of qubits. Importantly, the results of calculation do not depend on energy (entering through factor ६). In other words, all qubits give equal contributions to conductance and polarization. 

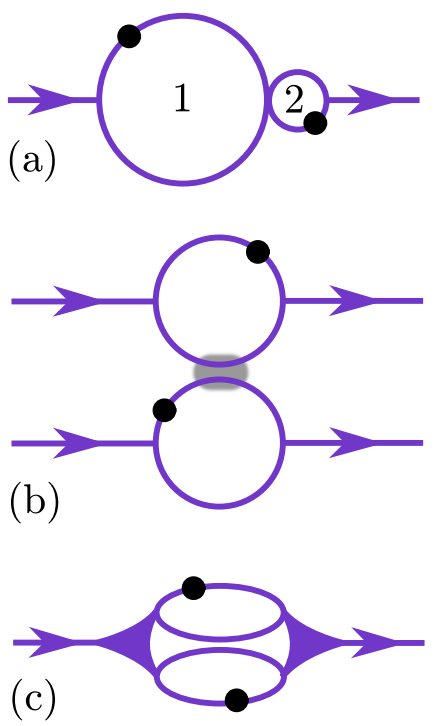

Fig. 7 Setups for one- and two-qubit operations. a Setup for onequbit operations based on interferometers of different sizes connected in series. The interferometer of larger size is more sensitive to the magnetic field and is used to manipulate the qubit spin state in the interferometer of smaller size (see also Fig. 8). b Setup for two-qubit operation based on interferometers connected in parallel and coupled by electron-electron interaction (the interaction region is marked by gray color). c Setup for creation of the pure state of arbitrary polarization with two interferometers containing strong impurities that block transmission through corresponding shoulders of each interferometer and with joint contacts to metallic leads allowing for coherent tunneling to both interferometers.

Using Eq. (25), for small $\theta, \lambda$, and $\phi$, we get

$\mathcal{T} \approx \lambda-\frac{\lambda^{3} \theta^{2}}{4 \lambda^{2}+\theta^{2}+4 \pi^{2} \phi^{2}}, P_{z} \approx-\frac{\lambda \theta^{2}}{4 \lambda^{2}+\theta^{2}+4 \pi^{2} \phi^{2}}$

The same equations are valid for $\phi$ close to $1 / 2$ with the replacement $\phi \rightarrow \phi-1 / 2$. One can check by direct calculation that dependence on $\xi$, and, consequently, on energy drops out after taking trace in Eq. (27). We see that the approach based on qubit representation not only reproduces results obtained by direct summation of the amplitudes within $\theta^{2}$ precision but also allows one to perform non-perturbative summation over relevant scattering processes and to get $\theta^{2}$ in the denominator of Eq. (30). Sharp dependence of $\mathcal{T}$ and $P_{z}$ on $\phi$ reflects tunability of the ensemble of qubits by an external magnetic field.

It is interesting to discuss possible generalizations of the hightemperature computing schemes to more complex systems involving several interferometers based on HES or HES arrays. (Experimental study of HES arrays has recently begun ${ }^{71}$.) The simplest examples of setups with two interferometers are shown in Fig. 7. Figure 7a schematically depicts two interferometers tunnel-connected in series to leads and to each other, with different edge lengths $L_{1}$ and $L_{2}\left(L_{1} \gg L_{2}\right)$. In the absence of a magnetic field, level spacings in these interferometers are very different: $\Delta_{1} \propto 1 / L_{1} \ll \Delta_{2} \propto 1 / L_{2}$. Then, for $\Delta_{1} \ll T \ll \Delta_{2}$, there are $T /$ $\Delta_{1}$ active qubits in the first interferometer and a single active qubit in the second one (see Fig. 8). On the other hand, for weak impurities, the spacing between qubit's levels is much larger in the first interferometer, $2 \Delta_{1} \phi_{1} \propto L_{1} \gg 2 \Delta_{2} \phi_{2} \propto L_{2}$ (here, we assume that homogeneous magnetic field is applied to both systems, so $\left.\phi_{1,2} \propto L_{1,2}^{2}\right)$. Hence, the first interferometer is much more sensitive to the magnetic field. In particular, one can tune an energy level in system 1 to be in resonance with the levels of an active qubit in

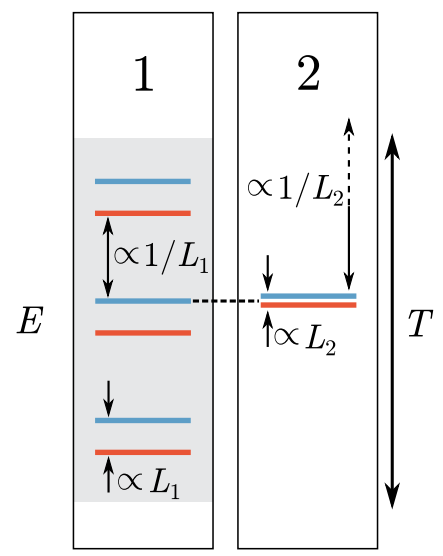

Fig. 8 Resonant coupling of two interferometers. Energy levels in a setup with two interferometers of different sizes $\left(L_{1}>L_{2}\right)$ connected in series (see Fig. 7a). Energy levels in the interferometer of larger size are much more sensitive to the magnetic field and can be tuned to manipulate a polarization of a single active qubit in the interferometer of smaller size.

system 2. Then, one can change the pure quantum state of the qubit 2 by a very small variation of the external field.

Similar to the low-temperature case ${ }^{34-38}$, one can suggest twoqubit manipulation schemes taking into account the electron-electron interaction. To this end, one can use interferometers connected in parallel and coupled by interaction (see Fig. 7b). The most essential feature of the high-temperature case distinguishing it from the low-temperature one is that now effective manipulation is possible for the whole ensemble of qubits. In particular, the simplest capacitive interaction between two interferometers would lead to the respective interactioninduced phase shift between states in the upper and down systems.

Finally, one can construct a setup for the creation of an outgoing polarized state with arbitrary polarization direction by using two interferometers containing strong impurities that block transmission through corresponding shoulders of each interferometer and with joint contacts to metallic leads allowing for coherent tunneling to both interferometers (see Fig. 7c). Assuming that unpolarized electrons enter the system from the left contact, we find that at the right contact there is the interference of two pure coherent states with various (in general, arbitrary) polarizations. As a result, outgoing electrons will be polarized with the direction different from the outgoing polarization of each interferometer.

\section{DISCUSSION}

We have studied coherent spin transport through HES of 2D topological insulators. We have shown that an unpolarized incoming electron beam entering the HES through one of the metallic leads acquires a finite polarization after transmission through the setup containing magnetic impurities. The finite polarization appears even in the fully classical regime and is therefore robust to dephasing. There also exists quantum contribution which survives at relatively high temperature and is tunable by magnetic flux piercing the area encompassed by HES. Specifically, the quantum contribution shows sharp identical $A B$ resonances as a function of magnetic flux with maxima (in the absolute value) at integer and half-integer values of the flux. For the setup with a single strong magnetic impurity blocking the transmission in one shoulder of $A B$ interferometer, and for large tunneling coupling, the spin polarization of transmitted electrons can achieve $100 \%$, which implies that outgoing electrons are in the pure quantum spin state. Also, this means that polarization 
can be transferred over distances on the order of the system size. The polarization reverses sign when an impurity is moved from one shoulder of the interferometer to another.

We discuss the possible application of obtained results for quantum computing. We demonstrate that tunneling interferometer based on HES can be described in terms of an ensemble of flux-tunable qubits giving equal contributions to conductance and spin polarization. Specifically, in presence of magnetic impurities and magnetic field, the initially doubly degenerate HES spectrum is split so that the appearing pairs of quantum states act as qubits with the spin orientation easily tuned by magnetic flux. The number of active qubits participating in the charge and spin transport is given by the ratio of the temperature and the level spacing. The interferometer can effectively operate at high temperatures and can be used for quantum calculations. In particular, the ensemble of qubits can be described by a single flux-tunable Hadamard operator. These findings are not sensitive to details of the system such as the geometry of the HES and allow one to speak about single-qubit operations such as X or Z-gate. Since we also predict the polarized state after passing the $A B$ interferometer by the unpolarized beam, we can prepare the qubits in the desired states.

If one uses the outgoing polarized state as the input for the next $A B$ interferometer, then one can further manipulate the states of the qubits. By arranging the setups involving several interferometers of certain geometries, we can produce non-trivial two-qubit operations needed for quantum computations. The obtained results open a wide avenue for applications in the area of quantum computing.

\section{METHODS}

Transitions through energy levels of a closed ring

Here, we derive analytical expressions describing anticrossing of quantum levels of right- and left-moving electrons on the example of a single impurity placed in the upper shoulder. We consider interferometer with the lengths of the upper and lower shoulders given by $s$ and $L-S$, respectively. The magnetic impurity is placed at position $x_{0}$ such that $0<$ $x_{0}<s$. Using the expression for scattering matrix (3), one can easily find the transfer matrix of impurity

$\hat{W}=\frac{e^{-i a}}{\cos \theta}\left(\begin{array}{cc}1 & i \sin \theta e^{-i \xi} \\ -i \sin \theta e^{i \xi} & 1\end{array}\right)$,

where $\xi=\varphi+2 k x_{0}$. In this section, we may add the constant value of forwarding scattering phase $a$ to the flux $2 \pi \phi$ and set $a=0$ below. The solution of the scattering problem for the electron with momentum $k$ on the whole system yields

$$
\left(\begin{array}{c}
a^{\uparrow} \\
a^{\downarrow}
\end{array}\right)=\hat{t}\left(\begin{array}{l}
b^{\uparrow} \\
b^{\downarrow}
\end{array}\right)
$$

where $\left(b^{\uparrow}, b^{\downarrow}\right)$ and $\left(a^{\uparrow}, a^{\downarrow}\right)$ are the amplitudes of incoming (from the left contact) and outgoing (to the right contact) waves and

$$
\hat{t}=\left(1-e^{-2 \lambda}\right) e^{2 \pi i \phi s / L}\left(\begin{array}{cc}
e^{i k s} & 0 \\
0 & e^{-i k s}
\end{array}\right) \times\left(\begin{array}{cc}
1 & 0 \\
0 & e^{\lambda}
\end{array}\right) \hat{g}\left(\begin{array}{cc}
1 & 0 \\
0 & e^{\lambda}
\end{array}\right),
$$

where

$\hat{g}=\frac{1}{1-\hat{W} \hat{\Lambda}} \hat{W}\left(\begin{array}{cc}1 & 0 \\ 0 & -1\end{array}\right)$,

$\hat{\Lambda}=\left(\begin{array}{cc}e^{i(Q L+2 \pi \phi)} & 0 \\ 0 & e^{i(-Q L+2 \pi \phi)}\end{array}\right)$,

and $Q$ is found from the condition $t^{2} e^{i k L}=e^{i Q L}$, yielding

$Q=k+i \frac{2 \lambda}{L}$.

The transmission coefficient and the spin polarization are expressed in terms of the matrix $\hat{t}$ as follows

$\mathcal{T}=\frac{1}{2}\left\langle\operatorname{Tr}\left(\hat{t} \hat{t}^{\dagger}\right)\right\rangle_{\epsilon}$

$\mathcal{P}=\frac{1}{2 \mathcal{T}}\left\langle\operatorname{Tr}\left(\hat{t} \sigma_{z} \hat{t}^{\dagger}\right)\right\rangle_{\epsilon}$

where $\langle\ldots\rangle_{e}$ stands for thermal averaging. Here, we neglect the Rashba coupling and assume that the incoming electrons are unpolarized. The matrix $\hat{g}$ can be presented as follows

$\hat{g}=\cos \theta\left[\frac{\hat{P_{1}}}{1-e^{i\left(Q L+2 \pi \phi_{0}\right)}}+\frac{\hat{P_{2}}}{1-e^{i\left(Q L-2 \pi \phi_{0}\right)}}+\left(\begin{array}{cc}0 & 0 \\ 0 & -1\end{array}\right)\right]$,

where $\phi_{0}$ is found from

$\cos \left(2 \pi \phi_{0}\right)=\cos \theta \cos (2 \pi \phi)$

and

$$
\begin{aligned}
\hat{P}_{1}= & \frac{1}{2 i \sin \left(2 \pi \phi_{0}\right) \cos \theta} \\
& \times\left(\begin{array}{cc}
-e^{-2 i \pi \phi}+e^{2 i \pi \phi_{0}} \cos \theta & i e^{-i(\xi+2 \pi \phi)} \sin \theta \\
i e^{i(\xi-2 \pi \phi)} \sin \theta & e^{-2 i \pi \phi}-e^{-2 i \pi \phi_{0}} \cos \theta
\end{array}\right), \\
\hat{P}_{2}= & -\frac{1}{2 i \sin \left(2 \pi \phi_{0}\right) \cos \theta} \\
& \times\left(\begin{array}{cc}
-e^{-2 i \pi \phi}+e^{-2 i \pi \phi_{0}} \cos \theta & i e^{-i(\xi+2 \pi \phi)} \sin \theta \\
i e^{i(\xi-2 \pi \phi)} \sin \theta & e^{-2 i \pi \phi}-e^{2 i \pi \phi_{0}} \cos \theta
\end{array}\right) .
\end{aligned}
$$

These are projection operators obeying:

$\hat{P}_{1}^{2}=\hat{P}_{1}, \hat{P}_{2}^{2}=\hat{P}_{2}, \hat{P}_{1} \hat{P}_{2}=0, \hat{P}_{1}+\hat{P}_{2}=1$.

Hadamard operator

Using Eq. (43), we can introduce the Hadamard operator

$\hat{H}=\hat{P}_{1}-\hat{P}_{2}$,

which obeys the standard property $\hat{H}^{2}=1$. However, in contrast to the conventional case, we have $H \neq H^{\dagger}$. We can now write

$\hat{P}_{1}=\frac{1+\hat{H}}{2}, \hat{P}_{2}=\frac{1-\hat{H}}{2}$

Thus defined Hadamard operator describes the isolated system and does not contain any information about tunneling coupling. We use now the following identities valid for arbitrary complex number $z$ with $\operatorname{Im} z>0$ and arbitrary $X \in[0,1)$ :

$\frac{e^{i \chi z}}{1-e^{i z}}=\left\{\begin{array}{l}i \sum_{n=-\infty}^{\infty} \frac{e^{2 \pi i x n}}{z-2 \pi n}, \text { for } 0<\chi<1, \\ i \sum_{n=-\infty}^{\infty} \frac{1}{z-2 \pi n}+\frac{1}{2}, \text { for } X=0\end{array}\right.$

Using Eqs. (45) and (46), we get

$\hat{g}=\frac{i \cos \theta}{2}\left[-i \hat{\sigma}_{z}+\frac{\Delta}{2 \pi} \sum_{n, a} \frac{1+a \hat{H}}{\epsilon-\epsilon_{n}^{a}+i \gamma / 2}\right]$,

with $\gamma=4 \lambda v_{\mathrm{F}} / L=2 \lambda \Delta / \pi$.

\section{Wave functions}

For completeness, we provide here the explicit form of wave functions for the energy levels (15)

$\psi_{n}^{ \pm}(x)=\frac{1}{\sqrt{\left|A^{ \pm}\right|^{2}+\left|B^{ \pm}\right|^{2}}}\left[\begin{array}{c}e^{i k_{n}^{ \pm}\left(x-x_{0}\right)} A^{ \pm} \\ e^{-i k_{n}^{ \pm}\left(x-x_{0}\right)} B^{ \pm}\end{array}\right]$.

Here, \pm labels energy levels [see Eq. (15)], $k_{n}^{ \pm}=\epsilon_{n}^{ \pm} / v_{\mathrm{F}}, A^{ \pm}=$ $\sin \theta e^{-i\left(\varphi \pm 2 \pi \phi_{0}\right)}, B^{ \pm}=\cos \theta \sin (2 \pi \phi) \pm \sin \left(2 \pi \phi_{0}\right)$, and $\varphi$ is the angle describing position of the magnetic moment of the impurity with a fixed projection on the local electron spin. Orthogonality condition reads

$\int_{0}^{L} d x\left\langle\psi_{n}^{\alpha}(x) \mid \psi_{m}^{\beta}(x)\right\rangle=\delta_{n m} \delta_{a \beta}, a, \beta= \pm$.

We emphasize that coefficients $A^{ \pm}$and $B^{ \pm}$that determines direction of local spin at $x=x_{0}$ do not depend on $n$. 
Received: 29 July 2019; Accepted: 23 October 2020; Published online: 13 November 2020

\section{REFERENCES}

1. National Academies of Sciences, E., Medicine, Grumbling, E. \& Horowitz, M. Quantum Computing: Progress and Prospects (The National Academies Press, Washington, DC, 2019).

2. Zwanenburg, F. A. et al. Silicon quantum electronics. Rev. Mod. Phys. 85, 961-1019 (2013).

3. Wolf, S. A. et al. Spintronics: a spin-based electronics vision for the future. Science 294, 1488-1495 (2001)

4. Žutić, I., Fabian, J. \& Das Sarma, S. Spintronics: fundamentals and applications. Rev. Mod. Phys. 76, 323-410 (2004).

5. Awschalom, D. D. \& Flatté, M. E. Challenges for semiconductor spintronics. Nat Phys. 3, 153-159 (2007)

6. Datta, S. \& Das, B. Electronic analog of the electro-optic modulator. Appl. Phys. Lett. 56, 665-667 (1990).

7. Crooker, S. A. et al. Imaging spin transport in lateral ferromagnet/semiconductor structures. Science 309, 2191-2195 (2005).

8. Appelbaum, I., Huang, B. \& Monsma, D. J. Electronic measurement and control of spin transport in silicon. Nature 447, 295-298 (2007).

9. Lou, X. et al. Electrical detection of spin transport in lateral ferromagnetsemiconductor devices. Nat. Phys. 3, 197-202 (2007)

10. Koo, H. C. et al. Control of spin precession in a spin-injected field effect transistor. Science 325, 1515-1518 (2009).

11. Kum, $\mathrm{H}$. et al. Room temperature single $\mathrm{GaN}$ nanowire spin valves with $\mathrm{FeCo} /$ MgO tunnel contacts. Appl. Phys. Lett. 100, 182407 (2012).

12. Wunderlich, J. et al. Spin Hall effect transistor. Science 330, 1801-1804 (2010).

13. Betthausen, $C$. et al. Spin-transistor action via tunable landau-zener transitions Science 337, 324-327 (2012).

14. Schmidt, G., Ferrand, D., Molenkamp, L., Filip, A. \& van Wees, B. Fundamental obstacle for electrical spin injection from a ferromagnetic metal into a diffusive semiconductor. Phys. Rev. B 62, R4790-R4793 (2000).

15. An, X.-T., Zhang, Y.-Y., Liu, J.-J. \& Li, S.-S. Spin-polarized current induced by a local exchange field in a silicene nanoribbon. New J. Phys. 14, 083039 (2012).

16. An, X.-T., Zhang, Y.-Y., Liu, J.-J. \& Li, S.-S. Measurable spin-polarized current in twodimensional topological insulators. J. Phys. Condens. Matter 24, 505602 (2012).

17. Michetti, P. \& Recher, P. Bound states and persistent currents in topological insulator rings. Phys. Rev. B 83, 125420 (2011).

18. Battilomo, R., Scopigno, N. \& Ortix, C. Spin field-effect transistor in a quantum spin-Hall device. Phys. Rev. B 98, 075147 (2018).

19. Zare, M. Resonance spin-transfer torque in ferromagnetic/normal-metal/ferromagnetic spin-valve structure of topological insulators. J. Magn. Magn. Mater 492, 165605 (2019).

20. Wójcik, P., Adamowski, J., Wołoszyn, M. \& Spisak, B. J. Intrinsic oscillations of spin current polarization in a paramagnetic resonant tunneling diode. Phys. Rev. B 86, 165318 (2012).

21. Slobodskyy, A. et al. Voltage-controlled spin selection in a magnetic resonant tunneling diode. Phys. Rev. Lett. 90, 246601 (2003)

22. Hauptmann, J. R., Paaske, J. \& Lindelof, P. E. Electric-field-controlled spin reversal in a quantum dot with ferromagnetic contacts. Nat. Phys. 4, 373-376 (2008).

23. Folk, J. A., Potok, R. H., Marcus, C. H. \& Umansky, V. A gate-controlled bidirectional spin filter using quantum coherence. Science 299, 679-682 (2003).

24. Wójcik, P., Adamowski, J., Wołoszyn, M. \& Spisak, B. J. Spin splitting generated in a Y-shaped semiconductor nanostructure with a quantum point contact. J. Appl. Phys. 118, 014302 (2015)

25. Matityahu, S., Aharony, A., Entin-Wohlman, O. \& Balseiro, C. A. Spin filtering in allelectrical three-terminal interferometers. Phys. Rev. B 95, 085411 (2017).

26. Shmakov, P. M., Dmitriev, A. P. \& Kachorovskii, V. Y. High-temperature AharonovBohm-Casher interferometer. Phys. Rev. B 85, 75422 (2012).

27. Tsai, W. F. et al. Gated silicene as a tunable source of nearly $100 \%$ spin-polarized electrons. Nat. Commun. 4, 1500 (2013).

28. Debray, P. et al. All-electric quantum point contact spin-polarizer. Nat. Nanotechnol. 4, 759-764 (2009).

29. Das, P. P. et al. Influence of surface scattering on the anomalous conductance plateaus in an asymmetrically biased $\ln \mathrm{As} / \mathrm{In} 0.52 \mathrm{Al} 0.48 \mathrm{As}$ quantum point contact. Nanotechnology 23, 215201 (2012).

30. Bhandari, N. et al. Steps toward an all-electric spin valve using side-gated quantum point contacts with lateral spin-orbit coupling. Adv. Nat. Sci.: Nanosci. Nanotechnol. 4, 013002 (2013).

31. Kohda, M. et al. Spin-orbit induced electronic spin separation in semiconductor nanostructures. Nat. Commun. 3, 1082 (2012).
32. Chuang, P. et al. All-electric all-semiconductor spin field-effect transistors. Nat Nanotechnol. 10, 35-39 (2015).

33. Földi, P., Molnár, B., Benedict, M. G. \& Peeters, F. M. Spintronic single-qubit gate based on a quantum ring with spin-orbit interaction. Phys. Rev. B 71, 033309 (2005).

34. Chen, W., Xue, Z.-Y., Wang, Z., Shen, R. \& Xing, D. Y. Quantum computing through electron propagation in edge states of quantum spin Hall systems. Eur. Phys. J. $B$ 87, 57 (2014).

35. Bautze, T. et al. Theoretical, numerical, and experimental study of a flying qubit electronic interferometer. Phys. Rev. B 89, 125432 (2014).

36. Bäuerle, C. et al. Coherent control of single electrons: a review of current progress. Reports Prog. Phys. 81, 056503 (2018).

37. Bordone, P., Bellentani, L. \& Bertoni, A. Quantum computing with quantum-Hall edge state interferometry. Semicond. Sci. Technol. 34, 103001 (2019).

38. Bellentani, L., Forghieri, G., Bordone, P. \& Bertoni, A. Two-electron selective coupling in an edge-state based conditional phase shifter. Phys. Rev. B 102 035417 (2020).

39. Stühler, R. et al. Tomonaga-Luttinger liquid in the edge channels of a quantum spin Hall insulator. Nat. Phys. 16, 47-51 (2020).

40. Strunz, J. et al. Interacting topological edge channels. Nat. Phys. 16, 83-88 (2020).

41. Dmitriev, A. P., Gornyi, I. V., Kachorovskii, V. Y. \& Polyakov, D. G. Aharonov-Bohm conductance through a single-channel quantum ring: persistent-current blockade and zero-mode dephasing. Phys. Rev. Lett. 105, 036402 (2010).

42. Kane, C. L. \& Mele, E. J. Quantum spin Hall effect in graphene. Phys. Rev. Lett. 95, 226801 (2005).

43. Bernevig, B. A., Hughes, T. L. \& Zhang, S. C. Quantum spin Hall effect and topological phase transition in HgTe quantum wells. Science 314, 1757-1761 (2006).

44. König, M. et al. Quantum spin Hall insulator state in HgTe quantum wells. Science 318, 766-770 (2007).

45. Roth, A. et al. Nonlocal transport in the quantum spin Hall state. Science $\mathbf{3 2 5}$ 294-297 (2009).

46. Gusev, G. M. et al. Transport in disordered two-dimensional topological insulators. Phys. Rev. B 84, 121302 (2011).

47. Brüne, C. et al. Spin polarization of the quantum spin Hall edge states. Nat. Phys 8, 485-490 (2012)

48. Kononov, A. et al. Evidence on the macroscopic length scale spin coherence for the edge currents in a narrow HgTe quantum well. JETP Lett. 101, 814-819 (2015).

49. Hasan, M. Z. \& Kane, C. L. Colloquium. Rev. Mod. Phys. 82, 3045-3067 (2010).

50. Qi, X.-L. \& Zhang, S.-C. Topological insulators and superconductors. Rev. Mod. Phys. 83, 1057-1110 (2011).

51. Kurilovich, P. D., Kurilovich, V. D., Burmistrov, I. S. \& Goldstein, M. Helical edge transport in the presence of a magnetic impurity. JETP Lett. 106, 593-599 (2017).

52. Niyazov, R. A., Aristov, D. N. \& Kachorovskii, V. Y. Tunneling Aharonov-Bohm interferometer on helical edge states. Phys. Rev. B 98, 045418 (2018).

53. Konig, M. et al. Quantum spin Hall insulator state in HgTe quantum wells. Science 318, 766-770 (2007).

54. Knez, I., Du, R.-R. \& Sullivan, G. Evidence for helical edge modes in inverted InAs/ GaSb quantum wells. Phys. Rev. Lett. 107, 136603 (2011).

55. Wu, S. et al. Observation of the quantum spin Hall effect up to 100 kelvin in a monolayer crystal. Science 359, 76-79 (2018).

56. Reis, F. et al. Bismuthene on a SiC substrate: a candidate for a high-temperature quantum spin Hall material. Science 357, 287-290 (2017)

57. Li, G. et al. Theoretical paradigm for the quantum spin Hall effect at high temperatures. Phys. Rev. B 98, 165146 (2018).

58. Jagla, E. A. \& Balseiro, C. A. Electron-electron correlations and the Aharonov-Bohm effect in mesoscopic rings. Phys. Rev. Lett. 70, 639-642 (1993).

59. Shmakov, P. M., Dmitriev, A. P. \& Kachorovskii, V. Y. Aharonov-Bohm conductance of a disordered single-channel quantum ring. Phys. Rev. B 87, 235417 (2013).

60. Dmitriev, A. P., Gornyi, I. V., Kachorovskii, V. Y., Polyakov, D. G. \& Shmakov, P. M. High-temperature Aharonov-Bohm effect in transport through a single-channel quantum ring. JETP Lett. 100, 839-851 (2015).

61. Dmitriev, A. P., Gornyi, I. V., Kachorovskii, V. Y. \& Polyakov, D. G. Spin-charge separation in an Aharonov-Bohm interferometer. Phys. Rev. B 96, 115417 (2017).

62. Chu, R. L., Li, J., Jain, J. K. \& Shen, S. Q. Coherent oscillations and giant edge magnetoresistance in singly connected topological insulators. Phys. Rev. B 80, 81102 (2009).

63. Masuda, S. \& Kuramoto, Y. Interference effects of helical current: geometrydependent spin polarization of transmitted electrons. Phys. Rev. B 85, 195327 (2012).

64. Dutta, P., Saha, A. \& Jayannavar, A. M. Aharonov-Bohm effect in a helical ring with long-range hopping: Effects of Rashba spin-orbit interaction and disorder. Phys. Rev. B 94, 195414 (2016).

65. Björnson, K. \& Black-Schaffer, A. M. Solid-state Stern-Gerlach spin splitter for magnetic field sensing, spintronics, and quantum computing. Beilstein J. Nanotechnol. 9, 1558-1563 (2018). 
66. Zhou, J., Zhou, T., Cheng, S.-g, Jiang, H. \& Yang, Z. Engineering a topological quantum dot device through planar magnetization in bismuthene. Phys. Rev. $B$ 99, 195422 (2019).

67. Ronetti, F., Vannucci, L., Dolcetto, G., Carrega, M. \& Sassetti, M. Spinthermoelectric transport induced by interactions and spin-flip processes in two-dimensional topological insulators. Phys. Rev. B 93, 165414 (2016).

68. Ronetti, F. et al. Polarized heat current generated by quantum pumping in twodimensional topological insulators. Phys. Rev. B 95, 115412 (2017).

69. Schmidt, T. L., Rachel, S., von Oppen, F. \& Glazman, L. I. Inelastic electron backscattering in a generic helical edge channel. Phys. Rev. Lett. 108, 156402 (2012).

70. Kainaris, N., Gornyi, I. V., Carr, S. T. \& Mirlin, A. D. Conductivity of a generic helical liquid. Phys. Rev. B 90, 075118 (2014).

71. Maier, $\mathrm{H}$. et al. Ballistic geometric resistance resonances in a single surface of a topological insulator. Nat. Commun. 8, 2023 (2017).

\section{ACKNOWLEDGEMENTS}

The work was supported by the Russian Science Foundation (Grant No. 20-12-00147) and by the Foundation for the Advancement of Theoretical Physics and Mathematics "BASIS". Work in Poland was supported by the Foundation for Polish Science through the grant $M A B / 2018 / 9$ for CENTERA.

\section{AUTHOR CONTRIBUTIONS}

All three authors contributed equally to the calculations and writing of the paper.

\section{COMPETING INTERESTS}

The authors declare no competing interests.

\section{ADDITIONAL INFORMATION}

Supplementary information is available for this paper at https://doi.org/10.1038/ s41524-020-00442-z.

Correspondence and requests for materials should be addressed to R.A.N.

Reprints and permission information is available at http://www.nature.com/ reprints

Publisher's note Springer Nature remains neutral with regard to jurisdictional claims in published maps and institutional affiliations.

Open Access This article is licensed under a Creative Commons Attribution 4.0 International License, which permits use, sharing, adaptation, distribution and reproduction in any medium or format, as long as you give appropriate credit to the original author(s) and the source, provide a link to the Creative Commons license, and indicate if changes were made. The images or other third party material in this article are included in the article's Creative Commons license, unless indicated otherwise in a credit line to the material. If material is not included in the article's Creative Commons license and your intended use is not permitted by statutory regulation or exceeds the permitted use, you will need to obtain permission directly from the copyright holder. To view a copy of this license, visit http://creativecommons. org/licenses/by/4.0/

(c) The Author(s) 2020 